\title{
Improved Tracking by Mitigating the Influence of the Human Body
}

\author{
Jens Trogh*, David Plets*, Luc Martens* and Wout Joseph* \\ * Department of Information Technology, iMinds - Ghent University, Belgium, jens.trogh@intec.ugent.be
}

\begin{abstract}
This paper presents a location tracking system that improves its performance by mitigating the influence caused by the human body of the user being tracked. The presence of such a user will influence the signal path between a body-worn mobile device and a receiving node. This influence will vary with the user's location and orientation and, as a result, the performance will deteriorate. By making use of the user's orientation towards the fixed infrastructure nodes, the influence of the body can be explicitly compensated, hereby improving the tracking accuracy. The overall system performance is extensively verified with experiments on a building-wide testbed. Compensating for this human body shadowing results in a relative improvement of 23.6\%.
\end{abstract}

Index Terms-Localization, Tracking, Human Body Shadowing, Wireless Networks, Indoor Environment

\section{INTRODUCTION}

Indoor localization and tracking systems have gained huge interest because of the many context-aware applications that have emerged lately. These applications are situated in many domains e.g., healthcare, industrial, cultural sector, etc. Many localization systems already try to cope with performance deterioration caused by multipath fading and diffraction. However, an important factor is the influence caused by the human body itself. The presence of a user's body can block the line-of-sight (LoS) between a body-worn mobile device (or $t a g$ ) and a receiving node, and cause additional losses. These additional losses are currently not accounted for and will generally decrease the accuracy of received signal strength indicator (RSSI) based localization systems. This effect has already been noted in literature but is often still neglected [1] Many localization applications verify their developed system by stepwise moving a device placed on a tripod, hereby explicitly removing the human from the equation. Practical human tracking applications however, always imply the presence of a user's body. In this work, a novel approach to mitigate the human body shadowing is investigated. By making use of a human body loss model and taking into account the body-worn tag's orientation towards the fixed infrastructure, the user's influence can be explicitly compensated. The orientation of a user is determined without making use of the classical compass or gyroscope approaches but with a novel orientation estimator built on top of our tracking algorithm.

\section{RELATED WORK}

In [2], a body shadowing mitigation method is used on top of an RSSI-based Monte Carlo localization technique and they achieve meter scale accuracies for a wrist-worn personnel tracking tag. The shadowing caused by a user's body is mitigated by using LoS and non-line-of-sight (NLoS) channel models. A disadvantage of this approach is that it depends on a manual differentiation of the LoS conditions and separate measurements need to be conducted for each LoS condition. In [3], video cameras are used to detect the human orientation and an empirical compensation model is used to compensate for body electronic interference. In [4], multiple sensors are placed on a user and the measured power level values are used for estimating the position and orientation of a user in a single room. They present a theoretical procedure to evaluate the maximum attainable performance with RSSI. Their analysis is based on ray-tracing to compute a fine grid of RSSI values and a maximum-likelihood approach for localization. In [5], a fingerprinting system based on neural networks is used for indoor localization with Bluetooth devices. They used a compass module to provide information about the user's orientation which improves the selection of the most adequate neural network to use. The achieved results are highly accurate but a lot of training data is needed because for every user orientation a neural network needs to be trained. In [6], [7] the losses caused by a human body are used as an advantage, they ask the user to rotate in place, simulating the behavior of a directional antenna. This directional analysis technique is used to localize an outdoor access point (AP) in [6] and in [7] this is used to know your own location.

In our work, the user's orientation is estimated based on previous positions so there is no need for manual differentiation [2], extensive measurements [2], [5], video cameras [3] or orientation tracking sensors like accelerometers, gyroscopes and compasses [5]. The experimental validation is done at 2.4 GHz using ZigBee nodes, on a building-wide testbed, not limited to a single room or theoretical framework [4].

\section{OVERVIEW OF THE SYSTEM}

In this section, a complete overview of the system is given. First, the tracking algorithm that serves as a basis for this work is explained. Then, the impact of the human body on the received signal strength is verified. Next, the solution to mitigate this body shadowing effect is presented. A flow graph of the complete system can be found in Figure 1.

The body-worn tag broadcasts packets that are received by the APs (fixed infastructure). The measured RSSI values and the tag's position are passed to the compensation model. 


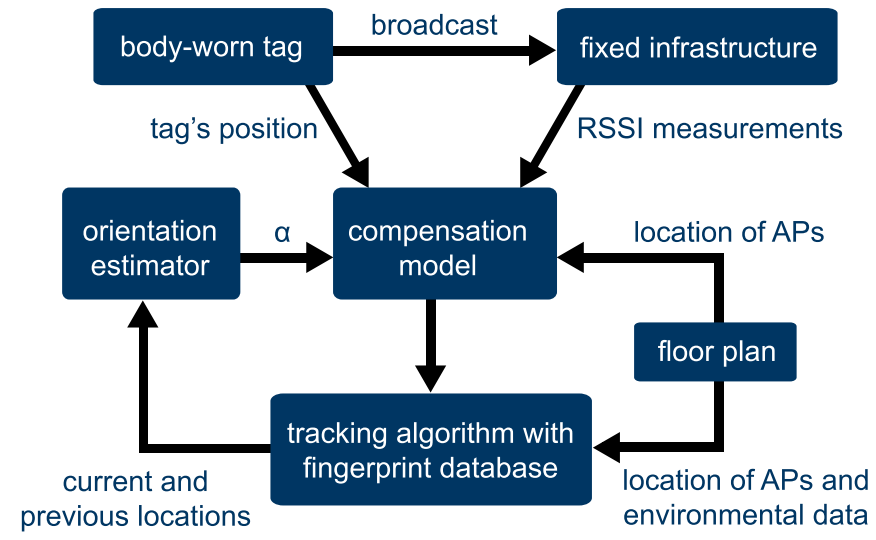

Figure 1: Flow graph of the system

This model uses the angle from the orientation estimator and the locations of the APs from the floor plan, to determine the influence caused by the user's body (Section III-C). The compensated RSSI values are passed to the tracking algorithm (Section III-A). This algorithm uses a fingerprint database and a floor plan (for environmental data and the locations of the APs), as additional inputs. The predicted current and previous locations are fed back to the orientation estimator to estimate the next orientation.

\section{A. Tracking algorithm}

A Viterbi-like tracking algorithm that uses off-the-shelf devices and the well-known RSSI fingerprinting technique serves as starting point for this work [8]. The environment of the user that is being tracked and a motion model are used as constraints to determine the most likely sequence of positions (path) instead of only the most likely current position (Viterbi principle). The following cost function is used to determine the most likely path:

$$
\text { Cost }_{i, T}=\sum_{t=1}^{T} \sum_{n=1}^{N}\left|R S S I_{t, n}^{\text {meas }}-R S S I_{t, n, i}^{r e f}\right|
$$

Cost $_{i, T}$ is the associated cost of the $i$ th path stored in memory at time step $T$ and $T$ is the number of time steps that went by since the tracking began. $N$ is the number of APs that measure the RSSI values from the packets broadcasted by the body-worn tag, $R S S I_{t, n}^{\text {meas }}$ is the RSSI measurement at time step $t$ from AP $n$ and $R S S I_{t, n, i}^{r e f}$ is the reference RSSI value from $\operatorname{AP} n$ for the position along path $i$ at time step $t$. The last position of the path with the lowest associated cost is taken as most likely current location. The calculations of paths and costs are not restarted every time a new measurement is received but the paths and costs from a previous iteration serve as input for the current iteration along with the new measurements. The reference RSSI values are stored in a fingerprint database and are derived from a path loss (PL) value calculated with a theoretical model from a network planner [9]:

$$
P L_{r e f}=\underbrace{P L_{0}+10 n \log _{10}\left(\frac{d}{d_{0}}\right)}_{\text {distance loss }}+\underbrace{\sum_{i} L_{W_{i}}}_{\text {cumulated wall loss }}+\underbrace{\sum_{j} L_{B_{j}}}_{\text {interaction loss }}
$$

$P L_{r e f}[\mathrm{~dB}]$ is the total path loss calculated with the theoretical model, $P L_{0}[\mathrm{~dB}]$ is the path loss at a reference distance $d_{0}[\mathrm{~m}], n[-]$ is the path loss exponent and $d[\mathrm{~m}]$ is the distance along the path between transmitter and receiver. The first two terms represent the path loss due to the traveled distance (distance loss), the third term (cumulated wall loss) is the sum of all wall losses $L_{W_{i}}$ when a signal propagates through a wall $W_{i}$ and the fourth term (interaction loss) takes into account the cumulated losses $L_{B_{j}}$ caused by all propagation direction changes $B_{j}$ of the propagation path from transmitter to receiver. Using a theoretical model avoids an expensive and time consuming measurement campaign but allows an immediate deployment at the expense of a slightly reduced accuracy.

\section{B. Problem: human body shadowing}

Human body shadowing occurs when the signal path between a body-worn mobile tag and a receiving node is completely or partly blocked. As already mentioned, the presence of a human body can influence the exchanged radio-frequency signals because of the large amount of water present in the human body (around 65\%). This influence manifests itself especially as a drop in signal strength; additional losses of around 10 to $30 \mathrm{~dB}$ are reported in literature [6]. To verify this effect, an experiment was conducted: a user was asked to turn $360^{\circ}$ around its axis, whilst wearing a mobile tag on his chest and back (see Figure $2 \mathrm{a}$ and $2 \mathrm{~b}$ ). Every 15 seconds the user turns $45^{\circ}$, taking 2 minutes for a full rotation. The RSSI values from the packets sent by the mobile tags are measured by forty fixed APs (the blue dots in Figure 3, for more details on this testbed, see Section IV). In Figure 4, the measured RSSI values as function of the user's orientation is shown for a nearby $(12 \mathrm{~m})$ and far away $(66 \mathrm{~m})$ AP. The location of the user and APs are indicated with a gold circle in Figure 3. An averaging window of 15 seconds was used.

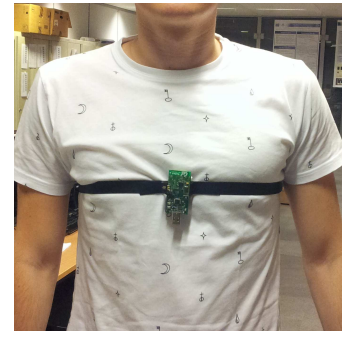

(a) Chest

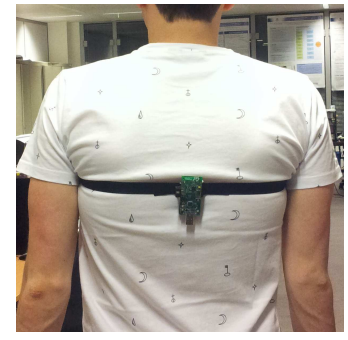

(b) Back
Figure 2: Body-worn tags (experiment)

From Figure 4, it is immediately clear that the orientation of a user has a significant influence on the measured RSSI values (on top of the variance caused by multipath). A high RSSI value measured by the chest tag corresponds almost 


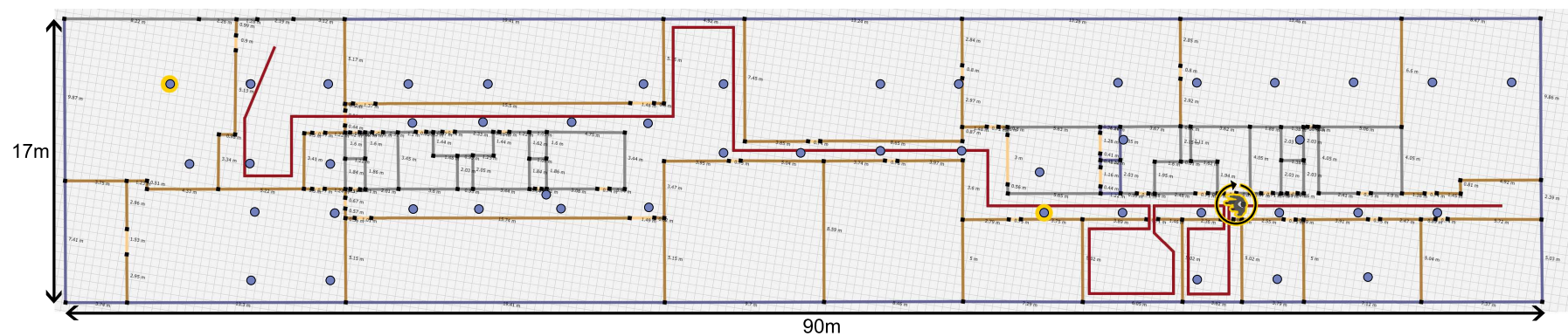

Figure 3: Testbed floor plan with locations of APs (blue), rotating user (yellow) and test trajectory (red)

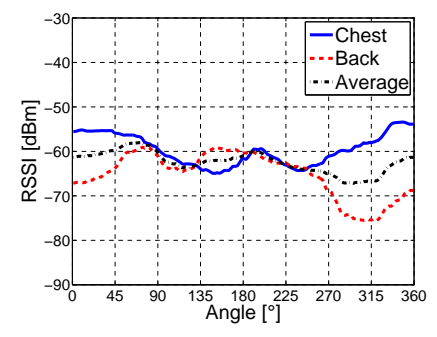

(a) Nearby AP (12 m)

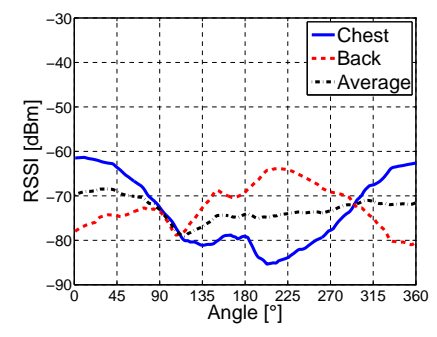

(b) Far away AP (66 m)

Figure 4: Human body shadowing while rotating a human body in an indoor environment

always to a low RSSI value measured by the back tag (and the other way around). This is because the human body is located between both body-worn tags and can e.g. block or attenuate the strongest path between the APs and mobile tags. The maximum differences between the chest and back mobile tag range from $1.8 \mathrm{~dB}$ to $25.2 \mathrm{~dB}$ with an average value of $14.8 \mathrm{~dB}$ (over all forty APs).

\section{Solution: compensating for user's orientation}

To mitigate the body shadowing, we will compensate explicitly for the influence caused by a user with a certain position and tag orientation towards the infrastructure APs. It consists of two parts: an orientation estimator and a compensation model.

1) Orientation estimation: Due to the nature of most building structures, people tend to walk in the same direction for at least a few seconds. This walking behavior can be exploited to estimate a user's orientation. More specifically, the angle between the previous and current location predictions can be used as an estimation for the next orientation. Such orientation estimator can be built on top of an existing tracking system which provides the current and previous positions as input. The next orientation is predicted as:

$$
\begin{gathered}
O_{t+1}=\frac{180}{\pi} \arctan \left(\frac{P_{t, y}-P_{t, y}^{a m}}{P_{t, x}-P_{t, x}^{a m}}\right) \\
P_{t}^{a m}=\frac{1}{K} \sum_{k=1}^{K} P_{t-k}
\end{gathered}
$$

$O_{t+1}\left[^{\circ}\right]$ is the next orientation, $P_{t}^{a m}$ is the arithmetic mean of $K$ previous predicted positions $P_{t}$ at time step $t$ and the $x$ and $y$ subscript indicate the $\mathrm{x}$ and $\mathrm{y}$ coordinate, respectively. The arctangent function with two arguments (atan2) is used to obtain the appropriate quadrant of the computed angle. The performance of this orientation estimator will depend on the accuracy of the tracking system itself and on the frequency of turns taken by a user. The robustness and responsiveness of the estimator are two contradictory demands. Including more previous location predictions (parameter $K$ from equation 4) will improve the accuracy of the estimator when few turns are present but this will rapidly decrease when a trajectory with more abrupt changes, is followed. The reason for the improvement is that the predicted locations are never completely accurate (e.g., variations around an actually followed straight line) and taking into account multiple positions can reduce the effect of prediction inaccuracies (averaging out the error). A simulation was performed to evaluate our estimator: two trajectories were outlined on a floor plan. The first trajectory has a length of $100 \mathrm{~m}$ and contains twenty-three $90^{\circ}$ turns and two $180^{\circ}$ turns, which results in an average of one turn every $4 \mathrm{~m}$ (red trajectory in Figure 5). The second one is a more straight trajectory, it has a length of $56 \mathrm{~m}$ and contains eight $90^{\circ}$ turns, which results in an average of one turn every $7 \mathrm{~m}$ (blue trajectory in Figure 5). The walking speed was set to $1 \mathrm{~m} / \mathrm{s}$.

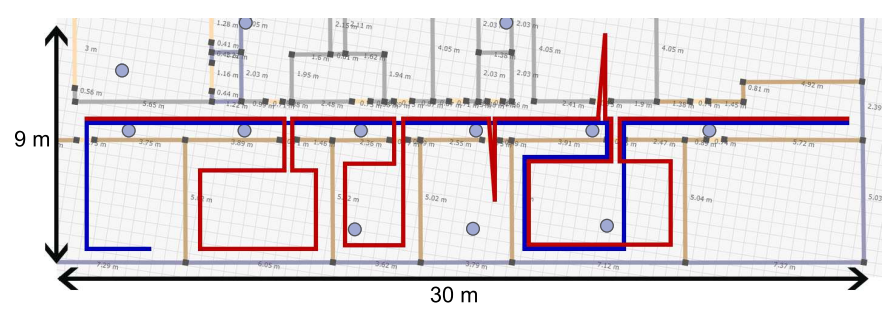

Figure 5: Red and blue trajectory for testing the orientation estimator (simulation)

The RSSI values corresponding to the positions along the trajectory were picked from the fingerprint database from Section III-A and are used as input for the tracking algorithm. Gaussian white noise with standard deviations of $1 \mathrm{~dB}, 3 \mathrm{~dB}$ and $6 \mathrm{~dB}$ was added to these RSSI values to simulate more realistic conditions. In Figure 6 the median values of the 
orientation estimation error are plotted as a function of the number of previous locations taken into account (parameter $K$ from equation 4 ). The simulations were repeated five times for averaging purposes.

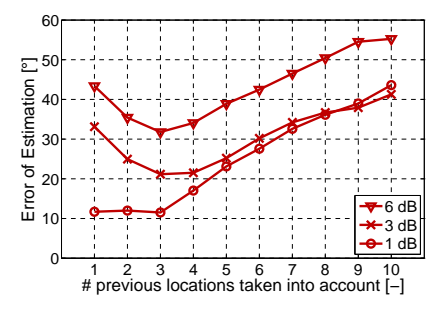

(a) Red trajectory

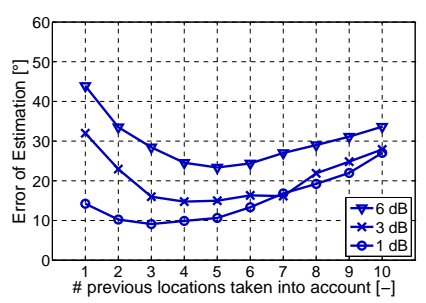

(b) Blue trajectory
Figure 6: Accuracy of orientation estimator

As expected, the median value of the error of estimation will first decrease when more previous positions are taken into account (averaging out inaccuracies) and will then increase again (due to turns taken). For the red trajectory (with many turns), the optimal value for $K$ is 3 with accuracies of $12^{\circ}$, $22^{\circ}$ and $32^{\circ}$ depending on amount of added noise. For the blue trajectory, this optimal value is 5 with accuracies of $10^{\circ}, 15^{\circ}$ and $22^{\circ}$ depending on amount of added noise. The user's orientation ranges from $0^{\circ}$ to $360^{\circ}$, meaning that an orientation error of $180^{\circ}$ is the worst possible result and that an error of $22^{\circ}$ corresponds to a relative error of $12 \%$. Because the followed trajectory is not known beforehand, the default $K$ value should not be set too high. A value between 3 and 5 is advised, this can still deal with more abrupt trajectory changes and is sufficiently accurate to compensate for the user's orientation (see Section V-A). Alternatively, an accelerometer, compass or gyroscope can be used to obtain a more precise orientation at the cost of additional required hardware.

Once the orientation of a user is known, the angles $\alpha$ between his mobile tag and the fixed infrastructure APs can be calculated (see Figure 7). It is assumed that the tag's position on the body and a floor plan with the locations of the fixed APs are known beforehand and that the user is walking forward.

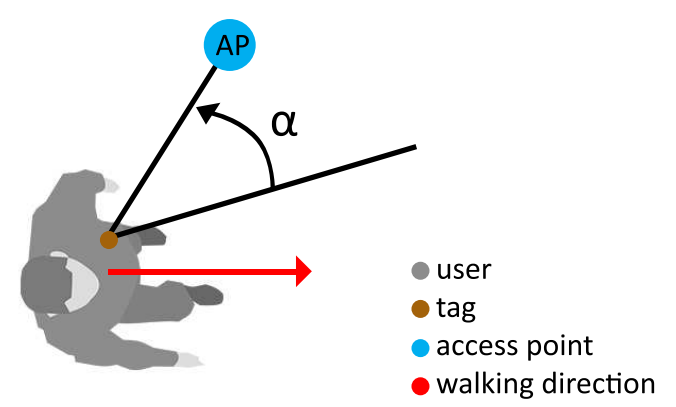

Figure 7: Angle between body-worn tag and AP (top view)

2) Compensation model: The orientation and angles between a user, his tag and all APs can now completely be characterized. Next, a compensation model is needed to estimate the influence due to the presence of this user. The mobile tag is calibrated based on the same measurements from Section III-B: the average value between the measurements and the RSSI fingerprinting database is taken as offset (calculated once). This means that the measurements will be an overestimation when tag and receiving node face each other directly $(\alpha=0)$ and be an underestimation when the human body is completely blocking the signal $(\alpha=\pi)$, see Figure 4 . A continuous model is chosen that uses the cosine of the angle $\alpha$ between the body-worn tag and an AP (see Figure 7), to calculate the compensation value:

$$
C_{\text {cosine }}(\alpha)=\frac{\text { compensation }}{2} \cdot \cos \alpha
$$

For this preliminary work, we have chosen this cosine model because it resemblances the measured RSSI values when a user turns around its axis (see Figure 4). Although, the influence of a user's body was up to $25 \mathrm{~dB}$ in our experiment from Section III-B, the default value of this compensation from Equation 5 is set to $6 \mathrm{~dB}$, which was found to be a good compromise between compensating for worst case body shadowing and when almost no shadowing is present (the maximum differences between the chest and back tag varied from $1.8 \mathrm{~dB}$ to $25.2 \mathrm{~dB}$, see Section III-B).

Including the human body shadowing compensation, results in following cost function for the tracking algorithm:

$$
\operatorname{Cost}_{i, T}=\sum_{t=1}^{T} \sum_{n=1}^{N}\left|\left(R S S I_{t, n}^{\text {meas }}-C_{\text {cosine }}\left(\alpha_{n}\right)\right)-R S S I_{t, n, i}^{\text {ref }}\right|
$$

$C_{\text {cosine }}\left(\alpha_{n}\right)$ is the cosine compensation model and uses the angle $\alpha_{n}$ between the body-worn tag and AP $n$ as input arguments. The other symbols are already defined for Equation 1 in Section III-A.

\section{EXPERIMENT CONFIGURATION}

The experiments are conducted on a wireless testbed, located on the third floor of an office building in Ghent, covering over $1500 \mathrm{~m}^{2}(17 \mathrm{~m}$ by $90 \mathrm{~m}$, see Figure 3$)$. It consists of several computer classes, offices and meeting rooms. The core is made of concrete walls, the inner structure is movable and made of layered drywall and the doors are made of wood. The wireless network consists of 48 fixed nodes that were installed at a height of $2.5 \mathrm{~m}$ (blue dots in Figure 3). A TelosB mote from Crossbow is used as body-worn mobile tag [10]. These are equipped with an embedded PIFA antenna and Chipcon CC2420 radio operating at $2.4 \mathrm{GHz}$ (IEEE 802.15.4/ZigBee compliant). There are 31 transmission power levels between -25 and $0 \mathrm{dBm}$ (set to $0 \mathrm{dBm}$ in all experiments). The mobile tag broadcasts 10 packets per second which are received by the infrastructure nodes and every second a location update is generated (the average RSSI values of the packets received within this second are used as input for the tracking algorithm). The test trajectory (indicated in red in Figure 3) has a total length of $140 \mathrm{~m}$, goes through three meeting rooms, a PC class and the hallway (remark: there are no APs installed in the second meeting room). The ground truth, i.e., the correct 
locations for comparison, are provided by fragmenting the test trajectory based on the number of location updates from an experiment (the user walked as continuously as possible with an average speed of $1.2 \mathrm{~m} / \mathrm{s}$ ).

\section{PERFormance EVAluation}

This section investigates the impact of body shadowing mitigation on the tracking accuracy. The presented results are obtained from experiments performed on the testbed described in Section IV.

\section{A. Impact of body shadowing mitigation}

To evaluate the performance when using the orientation estimator and compensation model, the test trajectory was repeated 5 times by a human whilst wearing a tag, placed on the central area of the chest (see Figure 2a). The Viterbi-like tracking algorithm is used to provide the previous positions that are needed to estimate the orientation (see Section III-C1). Three scenarios are considered: no compensation, compensation with the estimated orientation and compensation with the real orientation. The first scenario uses no compensation. The second and third scenario compensate for the influence of a user's body with the compensation model from Section III-C2. The only difference between them is that the second scenario uses the orientation estimator to determine the user's orientation, whereas the third scenario gets the real orientation as an additional input value. This is possible because the followed trajectory is known beforehand and it is assumed a user walks forward, hence the real orientation can be determined. These three scenarios are plotted for four different $K$ values: 1, 3, 5 and 7 (this parameter determines the number of previous locations taken into account to estimate the next user orientation, see Section III-C1). Thus, this will only affect the second scenario (compensation with estimated orientation).

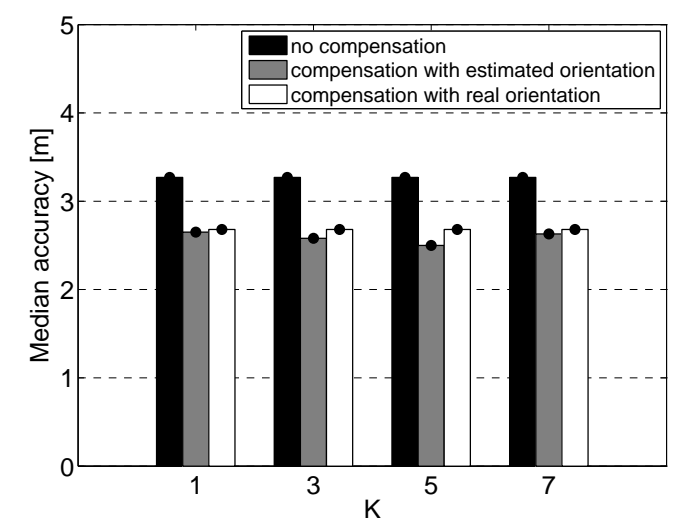

Figure 8: Impact of body shadowing mitigation

From Figure 8 it is clear that including the user's orientation to compensate for human body shadowing will always improve the tracking accuracy. Usage of our orientation estimator and compensation model results in additional improvements of
$18.9 \%, 21.1 \%, 23.6 \%$ and $19.6 \%$, for the four $K$ values, respectively. As expected, the optimal $K$ value is around 5 (with a median accuracy of $2.5 \mathrm{~m}$ ) but the difference remains rather limited. The median orientation error for this trajectory and $K$ value, averaged over the five runs, is $25^{\circ}$ (which is comparable to the simulations from Section III-C1). Furthermore, using the real orientation instead of the developed orientation estimator does not further improve the accuracy but performs similar. Normally, this real orientation is provided by a compass or gyroscope but for now this is accounted for by giving the real orientation as an additional input. Thus, using a compass or gyroscope has no added value in this case (body shadowing mitigation), but in other localization schemes they can be used as an additional feature to estimate the position itself, like e.g. dead reckoning, where their usage will be beneficial [11].

\section{CONCLUSIONS}

In this paper, a novel technique to mitigate the effects of body shadowing on a tracking algorithm's performance, is presented. By compensating explicitly for the body shadowing caused by the user that is being tracked, the tracking accuracy can be improved. This method uses a continuous compensation model and relies on the orientation of a user's tag towards the infrastructure nodes. The user's orientation is provided by an orientation estimator developed on top of a tracking algorithm. This alleviates the need for specialized hardware like a compass or gyroscope but achieves similar performance. Mitigating the human body shadowing results in a relative improvement of $23.6 \%$. Future work will include the investigation of optimal tag placement, usage of multiple body-worn tags simultaneously and methods to obtain location-dependent compensation models by making use of environmental and empirical data.

\section{ACKNOWLEDGMENT}

The authors would like to acknowledge the contributions of the COST IC1004 action.

\section{REFERENCES}

[1] Paramvir Bahl and Venkata N Padmanabhan. Radar: An in-building rfbased user location and tracking system. In INFOCOM 2000. Nineteenth Annual Joint Conference of the IEEE Computer and Communications Societies. Proceedings. IEEE, volume 2, pages 775-784. Ieee, 2000.

[2] William PL Cully, Simon L Cotton, William G Scanlon, and JB McQuiston. Body shadowing mitigation using differentiated los/nlos channel models for rssi-based monte carlo personnel localization. In Wireless Communications and Networking Conference (WCNC), 2012 IEEE, pages 694-698. IEEE, 2012.

[3] Xinfeng Li, Jin Teng, Qiang Zhai, Junda Zhu, Dong Xuan, Yuan F Zheng, and Wei Zhao. Ev-human: Human localization via visual estimation of body electronic interference. In INFOCOM, 2013 Proceedings IEEE, pages 500-504. IEEE, 2013.

[4] Paolo Barsocchi, Francesco Furfari, Paolo Nepa, and Francesco Potortì. Rssi localisation with sensors placed on the user. In Indoor Positioning and Indoor Navigation (IPIN), 2010 International Conference on, pages 1-6. IEEE, 2010.

[5] Marco Altini, Davide Brunelli, Elisabetta Farella, and Luca Benini. Bluetooth indoor localization with multiple neural networks. In Wireless Pervasive Computing (ISWPC), 2010 5th IEEE International Symposium on, pages 295-300. IEEE, 2010. 
[6] Zengbin Zhang, Xia Zhou, Weile Zhang, Yuanyang Zhang, Gang Wang, Ben Y Zhao, and Haitao Zheng. I am the antenna: accurate outdoor ap location using smartphones. In Proceedings of the 17th annual international conference on Mobile computing and networking, pages 109-120. ACM, 2011.

[7] Souvik Sen, Romit Roy Choudhury, and Srihari Nelakuditi. Spinloc: Spin once to know your location. In Proceedings of the Twelfth Workshop on Mobile Computing Systems \& Applications, page 12. ACM, 2012.

[8] Jens Trogh, David Plets, Luc Martens, and Wout Joseph. Advanced real-time indoor tracking based on the viterbi algorithm and semantic data. International Journal of Distributed Sensor Networks, 501:271818, 2015.

[9] David Plets, Wout Joseph, Kris Vanhecke, Emmeric Tanghe, and Luc Martens. Coverage prediction and optimization algorithms for indoor environments. EURASIP Journal on Wireless Communications and Networking, 2012(1):1-23, 2012.

[10] Crossbow TelosB Datasheet. http://www.willow.co.uk/TelosB Datasheet.pdf. Accessed: 2015-06-16.

[11] Moustafa Alzantot and Moustafa Youssef. Uptime: Ubiquitous pedestrian tracking using mobile phones. In Wireless Communications and Networking Conference (WCNC), 2012 IEEE, pages 3204-3209. IEEE, 2012. 